\title{
Efecto de la aplicación de acibenzolar-S-metil y aceite agrícola sobre la fitotoxicidad en plántulas de banano
}

\author{
Effect of the application of acibenzolar-S-methyl and agricultural \\ oil on phytotoxicity in banana seedlings
Rómulo García-Velasco ${ }^{1}$ (D), Analesa Olinda-Skeete ${ }^{2}$, Nury Pérez-Valdés², Barbarita Companioni-González ${ }^{3 *}$ [D

${ }^{1}$ Laboratorio de Fitopatología, Universidad Autónoma del Estado de México, km 1.5 Carretera Tenancingo-Villa Guerrero, 52400, Estado de México, México.

${ }^{2}$ Departamento de Ciencias Agropecuarias, Universidad de Ciego de Ávila "Máximo Gómez Báez", Carretera a Morón km 1.2, 69450, Ciego de Ávila, Cuba.

${ }^{3}$ Departamento de Horticultura, Universidad Autónoma Agraria Antonio Narro, Calzada Antonio Narro, No. 1923, Buenavista, 25315, Saltillo, Coahuila, México.

*Autor para correspondencia: bcompanioni2007@gmail.com

Fecha de recepción:

26 de abril de 2020

Fecha de aceptación:

27 de julio de 2020

Disponible en línea:

23 de octubre de 2020

Este es un artículo en acceso

abierto que se distribuye de acuerdo a los términos de la licencia Creative Commons.

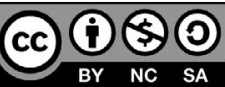

Reconocimiento-

NoComercial-

CompartirIgual 4.0

Internacional

\section{RESUMEN}

La aplicación de inductores de resistencia sistémica en cultivos de interés agrícola se ha convertido en una herramienta estratégica en el manejo de las enfermedades en los cultivos agrícolas. El presente trabajo se hizo con el objetivo de determinar el efecto de la aplicación de acibenzolar-S-metil, y el aceite agrícola sobre la fitotoxicidad en plántulas de banano. Se demostró que el aceite agrícola causó fitotoxicidad sobre las plántulas de banano, no así el producto inductor acibenzolar-S-metil.

\section{PALABRAS ClaVe}

Fitotoxicidad, marchitez por Fusarium, Musa.

\section{ABSTRACT}

Inducers of systemic resistance in plants have become a powerful tool for managing diseases in agricultural crops. This study was carried out in order to determine the effect of the application of acibenzolar-S-methyl, and agricultural oil on the phytotoxicity on banana seedlings. Agricultural oil was shown to cause phytotoxicity on banana seedlings, unlike the inductor acibenzolar-S-methyl, which makes the use of the latter suitable for the protection of banana seedlings.

\section{KEYWORDS}

Phytotoxicity, Fusarium wilt, Musa. 


\section{INTRODUCCIÓN}

Los bananos (Musa spp.) están entre los cultivos más importantes en los países del trópico y el subtrópico (FAO 2017). En Cuba, los bananos y plátanos constituyen uno de los principales cultivos para lograr el equilibrio de productos en el mercado, de ahí que se preste gran interés en lograr altos rendimientos y producciones de las plantaciones en explotación (López 2002). Sin embargo, la producción de estos cultivos se encuentra amenazada por el ataque de enfermedades como el Mal de Panamá o marchitez por Fusarium causada por Fusarium oxysporum f. sp. cubense (FOC) (Ploetz 2015). En los últimos años, existe un consenso general de que la única forma de control efectiva y sostenible para esta enfermedad es la resistencia del hospedero (Wu et al. 2010). Por ello, la aplicación de inductores de resistencia sistémica en cultivos de interés agrícola se ha convertido en una herramienta estratégica en el manejo de las enfermedades en los cultivos agrícolas, capaz de acelerar la respuesta de defensa de la planta al ataque del patógeno, así como de proporcionar una protección de larga duración, y disminuir la aplicación de plaguicidas químicos en el control de enfermedades, entre otras ventajas (Shahini et al. 2010). En este sentido, se han desarrollado varios productos compatibles con el ambiente y que inducen a la planta a activar sus mecanismos de defensa contra las enfermedades. Por otra parte, el aceite agrícola ayuda a la dispersión y penetración de estos productos en la hoja de la planta (Orozco et al. 2013). El presente trabajo se llevó a cabo con el objetivo de determinar el efecto de la aplicación de acibenzolar-S-metil y de aceite agrícola sobre la fitotoxicidad en plántulas de banano.

\section{Materiales y Métodos}

La investigación se desarrolló en el área de aclimatización del Centro de Bioplantas de la Universidad de Ciego de Ávila, Cuba. En la primera parte de la experimentación, se determinó el efecto de la aplicación foliar de diferentes dosis de la mezcla del producto inductor acibenzolar-S-metil (Bion ${ }^{\circledR} 50$ WG, SYNGENTA), sobre la fitotoxicidad en plántulas del cultivar Gros Michel inoculadas con Fusarium oxysporum f. sp. cubense, en condiciones de invernadero.
Para ello, se utilizaron plántulas enraizadas de banano con $10 \mathrm{~cm}$ de altura, las cuales fueron inoculadas a través del bioensayo de cortes de raíces y sumergidas en una suspensión de esporas de $8 \times 10^{5}$ conidios $\mathrm{mL}^{-1}$, durante 15 minutos. Posteriormente, se sembraron en bolsas de polietileno que contenían suelo ferralítico rojo y cachaza (1:1; v:v).

Se efectuaron aplicaciones foliares de diferentes dosis de la mezcla del producto inductor (acibenzolar-S-metil + aceite agrícola). Las dosis probadas fueron: 0,20 y $40 \mathrm{~g}$ de i. a. ha ${ }^{-1}$. El tratamiento control consistió en plántulas donde sólo se aplicó agua. Al cabo de treinta días de iniciar el experimento, se hicieron observaciones visuales de síntomas de fitotoxicidad en las hojas de las plántulas y de la severidad de la enfermedad a través de los síntomas internos en el pseudotallo. En la segunda parte de la experimentación, se probó el efecto de la aplicación foliar de cada componente de la mezcla del producto inductor en plántulas del cultivar Gros Michel. Se compararon los siguientes tratamientos: 1) plántulas aplicadas con agua (tratamiento control); 2) plántulas aplicadas con producto inductor (40 g i.a. ha- ${ }^{-1}$ de acibenzolar-S-metil); 3) plántulas aplicadas con aceite agrícola, y 4) plántulas aplicadas con mezcla del producto inductor (aceite agrícola $+40 \mathrm{~g}$ i.a. ha ${ }^{-1}$ de acibenzolar-S-metil). El procedimiento para la siembra de las plántulas del cultivar Gros Michel fue similar al descrito en el experimento anterior.

A los treinta días de la aplicación foliar de los diferentes tratamientos se procedió a la evaluación de los siguientes parámetros: número de hojas brotadas; número de hojas necrosadas; número de hojas muertas, y altura de la plántula $(\mathrm{cm})$. En todo el experimento se utilizó un diseño completo al azar, donde cada tratamiento incluyó 10 plántulas. En el procesamiento estadístico de los datos se utilizó el software Statistical Package for Social Sciences (SPSS para Windows, versión 8, Copyright SPSS Inc., 1989-1997).

\section{Resultados Y Discusión}

Después de los 5 días de la aplicación foliar de la mezcla del producto inductor, se observaron síntomas visibles de fitotoxicidad en las hojas de las plántulas de banano en las diferentes dosis probadas $(20$ y $40 \mathrm{~g}$ de i. a. ha-1 ${ }^{-1}$; no fue así en el tratamiento control (Figura 
1). Los síntomas de fitotoxicidad se manifestaron en el amarillamiento de las hojas, y después de treinta días se observaron quemaduras en las hojas. Esta fue la razón por la cual en el siguiente experimento se probó el efecto de cada componente de la mezcla del producto inductor de forma separada. Sin embargo, al determinar la severidad de la enfermedad a través de los síntomas internos en el pseudotallo, no se observaron síntomas de colonización u obstrucción de los tejidos vasculares de las plántulas. La temperatura y la humedad relativa durante los treinta días no fueron las favorables para el desarrollo de la enfermedad en las condiciones de invernadero. Los valores de temperaturas se comportaron en el rango de 29 a $40^{\circ} \mathrm{C}$, y la humedad relativa en el rango de 60 a $70 \%$. Los factores ambientales mencionados constituyeron limitantes para el buen desarrollo de la enfermedad. La temperatura y humedad relativa son unos de los principales factores que determinan el crecimiento y desarrollo de los hongos. Varios autores han planteado que las condiciones favorables para el desarrollo de marchitez por Fusarium en banano requieren de un rango de temperatura de 25 a $28^{\circ} \mathrm{C}$, y una humedad relativa alrededor de 80\% (González et al. 2012; Ploetz

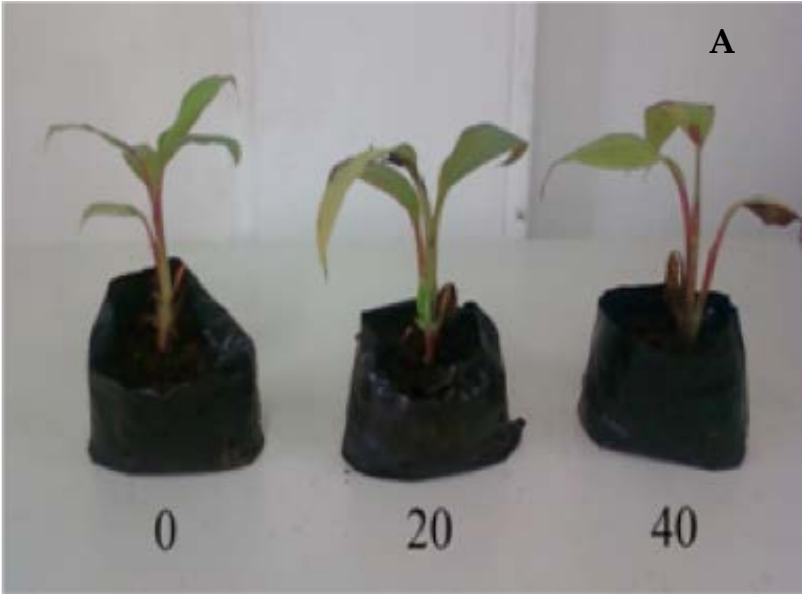

Plántulas a los 5 días de la aplicación de la mezcla del producto inductor. la aplicación foliar de $40 \mathrm{~g}$ de i.a. ha- ${ }^{-1}$ de acibenzolar-S-metil, con resultados similares en el tratamiento control (Figura 2 A y D), pero con marcadas diferencias significativas respecto a los tratamientos que contenían en su aplicación foliar aceite agrícola (Figura 2 B y C), donde se obtuvieron los mayores valores de número de hojas necrosadas $(85 \%)$ y número de hojas muertas (94\%). En este sentido, Poole et al. (2012) plantearon que el aceite agrícola derivado de la industria petrolera en Cuba constituye un excelente penetrante, razón por la cual puede causar fitotoxicidad (daño tisular).

\section{Conclusión}

El aceite agrícola causó fitotoxicidad sobre las plántulas de banano, no así el producto inductor acibenzolar-S-metil. De este modo, se continúan los experimentos encaminados a optimizar las condiciones para la aplicación del producto acibenzolar-S-metil como inductor abiótico en plántulas del cultivar Gros Michel en condiciones controladas de invernadero.

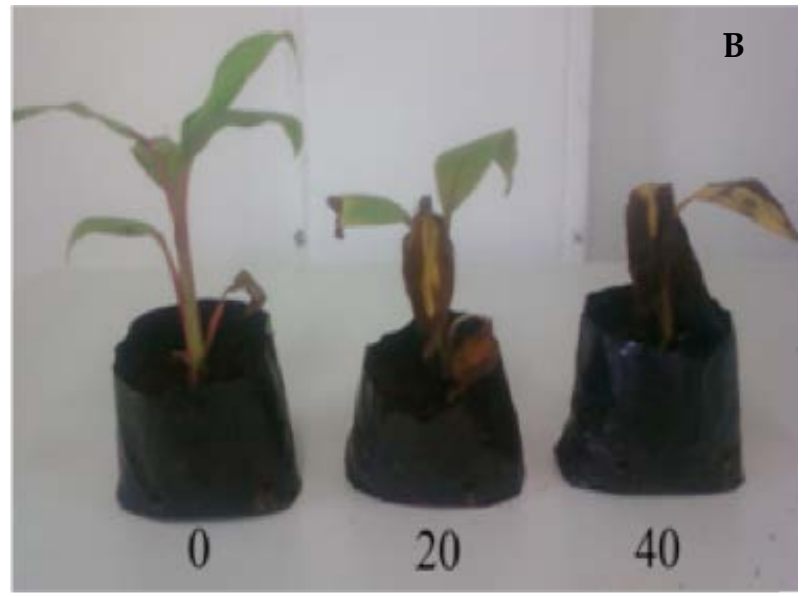

Plántulas a los 30 días de la aplicación de la mezcla del producto inductor.

Figura 1. Presencia de síntomas de fitotoxicidad; 5 días después de la aplicación 0,20 y $40 \mathrm{~g}$ i.a. ha-1 (A); y 30 días después de la aplicación 0 , 20 y 40 g i.a. ha-1 (B) en plántulas de banano inoculadas con Fusarium oxysporum f. sp. cubense raza 1.

2015).

Por otra parte, en el efecto de la aplicación foliar de los componentes de la mezcla del producto inductor en plántulas de banano se alcanzaron valores mayores de $80 \%$ en hojas brotadas, y en la altura de las plántulas, valores por encima de $30 \mathrm{~cm}$, en el tratamiento con 
A

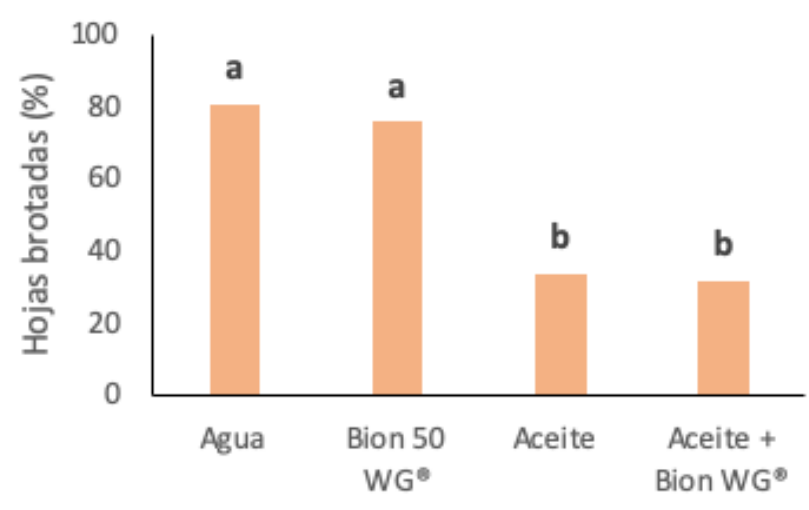

Aplicación foliar de los componentes de la mezcla del producto inductor

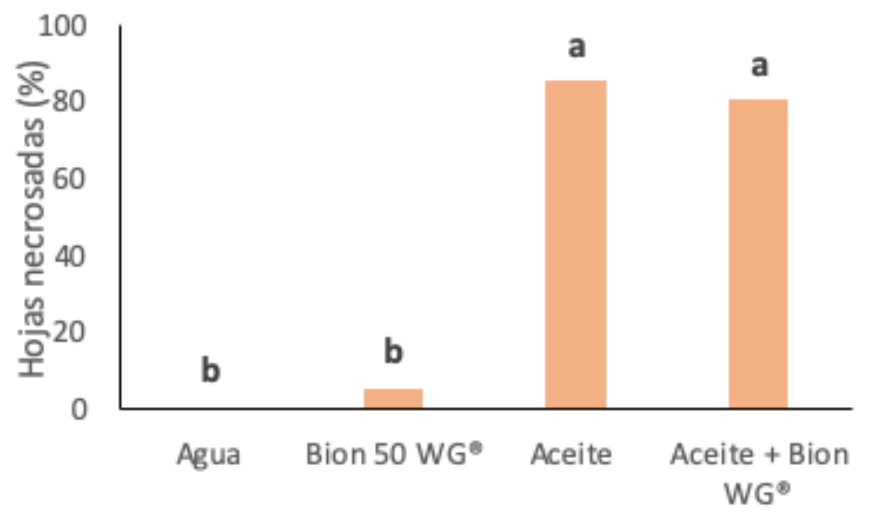

Aplicación foliar de los componentes de la mezcla del producto inductor

C

D

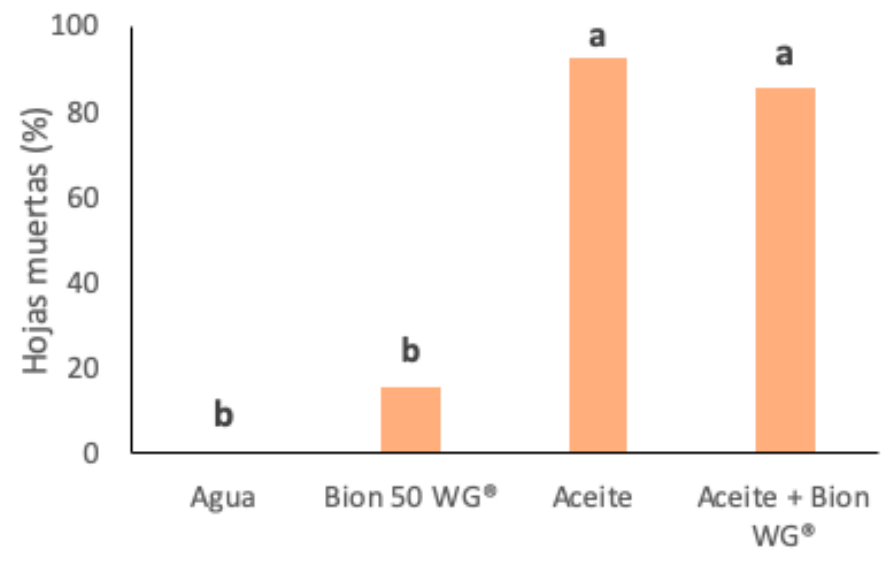

Aplicación foliar de los componentes de la mezcla del producto inductor

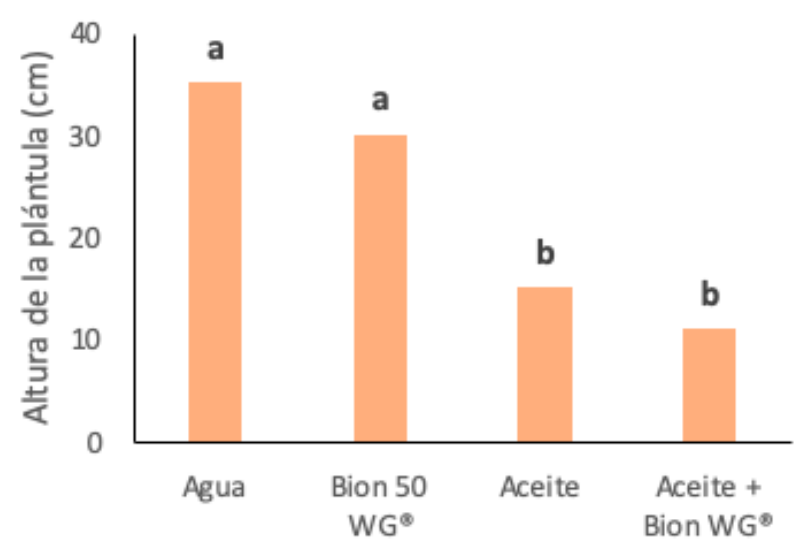

Aplicación foliar de los componentes de la mezcla del producto inductor

Figura 2. Efecto de la aplicación foliar de los componentes de la mezcla del producto inductor en plántulas de banano. Número de hojas brotadas (A); número de hojas necrosadas (B); número de hojas muertas (C); altura de la planta (D). En cada figura, medias con letras iguales indican que no existen diferencias estadísticamente significativas (ANOVA, Tukey, $p \leq 0.05$ ). 


\section{LITERATURA CITADA}

[FAO] Food and Agriculture Organization of the United Nations. [internet]. 2017. Global programme on banana Fusarium wilt disease: Protecting banana production from the disease with focus on tropical race 4 (TR4). FAO, Rome, ITA. [cited 2020 June]. Disponible en: https://www.fao.org/3/a-i7921e.pdf

González I, Arias Y, Peteira B. 2012. Aspectos generales de la interacción Fusarium oxysporum f. sp. lycopersici-tomate. Protección Vegetal 27: 1-7.

López J. 2002. Avances y perspectivas para el mejoramiento genético del banano (Musa spp.) por técnicas biotecnológicas y nucleares en el INIVIT. Infomusa 11: 18-20.

Orozco M, García K, Manzo G, Guzmán S, Martínez L, Beltrán M, Garrido E, Torres JA, Canto B. 2013. La Sigatoka negra y su manejo integrado en banano. Libro Técnico Número 1. Secretaría de Agricultura, Ganadería, Desarrollo Rural, Pesca y Alimentación, Instituto Nacional de Investigaciones Forestales Agrícolas y Pecuarias, Centro de Investigación Regional Pacífico Centro, Campo Experimental Tecomán. Tecomán, México.

Ploetz R. 2015. Management of Fusarium wilt banana: A review with special reference to tropical race 4 . Crop Protection 73: 7-15. https://doi.org/10.1016/j. cropro.2015.01.007

Poole GJ, Smiley RW, Paulitz TC, Walker CA, Carter AH, See DR, Garland-Campbell K. 2012. Identification of quantitative trait loci (QTL) for resistance to Fusarium crown rot (Fusarium pseudograminearum) in multiple assay environments in the Pacific Northwestern US. Theoretical and Applied Genetics 125: 91-107. https:// doi.org/10.1007/s00122-012-1818-6

Shahini FS, Keshavarzi M, Hassanzade N, Hashemi M, Abdollahi HY, Tawosi M. 2010. In vitro evaluation of acibenzolar-s-methyl on inhibition of fire blight in apple cv. golden delicious, Iran. Journal Plant Pathology 46(3): 77-79.

Wu YL, Yi GJ, Peng XX. 2010. Rapid screening of Musa species for resistance to Fusarium wilt in an in vitro bioassay. European Journal of Plant Pathology 128: 409-415. https://doi.org/10.1007/s10658-010-9669-y 